\title{
Virtual Host Network Traffic Scheduling Based on Composite Particle Swarm Optimization
}

\author{
Huan Wang, Jian Gu, Jianping Zhao*, Xiaoqiang Di, Zhanfang Chen, Mingquan E \\ and Bo Li \\ School of Computer Science and Technology, Changchun University of Science \\ and Technology, China \\ wanghuan273@126.com
}

\begin{abstract}
Aiming at solving the problems of unbalanced cloud data center virtual host network link bandwidth utilization and multi QoS (Quality of Service) security issues in different services, in this paper, a multi QoS based network traffic scheduling model for virtual machines is proposed. In order to achieve the global optimal network traffic scheduling, the model uses Shannon information entropy to measure the network link traffic balance and uses the linear weighted sum method to transform the multi objective optimization into a single objective problem. At the same time, we put forward a network traffic scheduling algorithm based on particle swarm composite. By dividing the original solution space into several equivalent subspaces, each particle swarm is independently searched in the subspace. Therefore, the algorithm has a faster convergence rate and avoids falling into the local optimal value problem easily caused by the traditional algorithm. The simulation results show that the model and algorithm can improve the network link bandwidth utilization and guarantee the synchronization requirements of high priority data.
\end{abstract}

Keywords: Bandwidth utilization; QoS; Composite particle swarm; Traffic scheduling.

\section{Introduction}

With the rapid development of virtualization technology, more and more data centers use virtualization technology to provide services and improve the utilization of physical resources. The virtual host has a high demand on the network during migration and operation. How to realize the optimize network traffic control between the virtual hosts has become one of the problems to be solved in the cloud data center. Virtual host network traffic optimization is an intelligent dynamic management of network bandwidth resources in the data center. Virtual host network traffic optimization can control the physical and virtual network data traffic effectively and enhance the link utilization.

Domestic and foreign researchers have conducted a lot of researches on network traffic control. Literature [1] has studied the QoS guarantee problem of cloud data center network virtualization and has proposed a kind of QoS guarantee mechanism. The network virtualization based on SDN technology is studied in the literature [2]. Literature [3] focused on a network bandwidth guarantee mechanism between multiple virtual machines. In literature [4], the network sharing mechanism among multiple data centers is studied, and the simulation is carried out. The traffic control problem based on session information feedback is studied in literature [5], and the stability of the network traffic control mechanism is proved. The fairness of the network traffic control problem is studied in literature [6], and the distributed traffic control algorithm based on the round trip time delay is given in the paper. A network flow control model and algorithm based on stochastic estimation is proposed in literature [7]. A network flow control algorithm based on bargaining is proposed in literature [8], and it is proved that the algorithm is 
stable and convergent. In literature [9], the control strategy of network traffic for large scale users is studied, and the existence of Nash equilibrium is proved.

Aiming at these problems, a multi QoS based network traffic scheduling model for multiple virtual machines is proposed. According to the characteristics of the hierarchical structure of the network, this paper proposed a kind of network traffic scheduling algorithm based on composite particle swarm optimization. The model can ensure the performance requirements of data transmission at different levels, and maximize the utilization of network link bandwidth resources among virtual machines.

\section{Network Traffic Scheduling Model}

Cloud data center is composed of virtual host, host node and network node and has the characteristics of hierarchy. The configuration of the virtual host is shown in Figure 1.

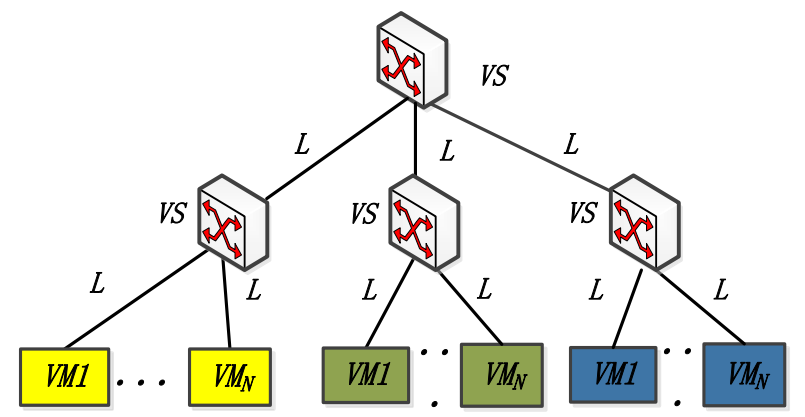

a) Virtual machine deployment logic diagram

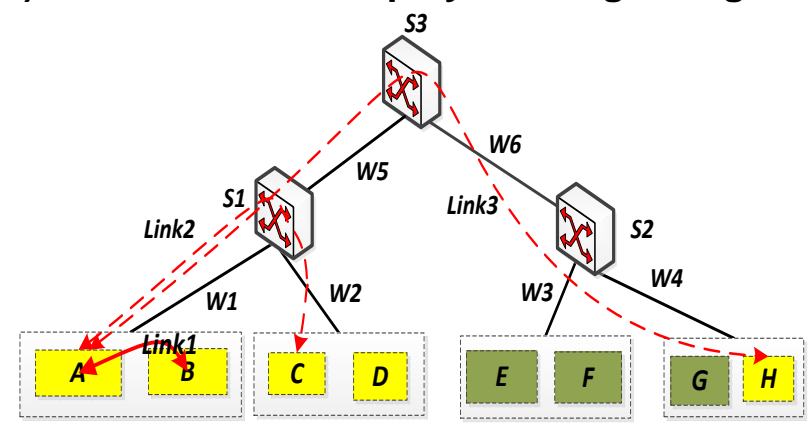

b) Virtual machine physical deployment diagram

Figure 1. The Configuration of the Virtual Host

According to the above network structure, the flow resource scheduling problem is formalized. Full duplex communication is used in the model. The interconnection between the host nodes uses an independent link for bidirectional transmission. We assume that the number of virtual hosts and network nodes in the cloud data center network is $T$, the number of physical link between host nodes is $W$. The number of data link between the virtual hosts is $G$. The definition of model is given as follow.

(1) Data link traffic

Let $W_{i j}$ be the physical link between the host node $i$ and the host node $j . G_{i j}$ is the traffic between the virtual machine on the host node $i$ and the virtual machine on the host node $j$. Link flow of a data link from node $i$ to node $j$ is $L_{i j}$. The segment vector formula of the data link is expressed as follows.

$$
G_{i j}=\left(L_{i x}, \ldots, L_{y j}\right) .
$$

Bandwidth utilization 
$W_{i j}$ is the sum of all data link flows for the host node $i$ to the host node $j$. Assuming the maximum bandwidth of the link is $B_{i j}$, the bandwidth utilization ratio $S_{i j}$ of the link $W_{i j}$ is:

(3) Flow matrix

$$
S_{i j}=\frac{W_{i j}}{B_{i j}}=\frac{\sum L_{i j}}{B_{i j}} .
$$

In the model, $\mathrm{QoS}=\{1,2,3\}$ is used to represent the data traffic assignment of different communication levels from the node $i$ to the node $j$, corresponding to the flow matrix $\left(M_{1, i j}\right)_{T \times T},\left(M_{2, i j}\right)_{T \times T},\left(M_{3, i j}\right)_{T \times T}$. By adding the traffic matrix of QoS level of these three kinds of communication, we can get the total traffic matrix of node from $i$ to $j$.

$$
\left(M_{i j}\right)_{T \times T}=\left(M_{1, i j}\right)_{T \times T}+\left(M_{2, i j}\right)_{T \times T}+\left(M_{3, i j}\right)_{T \times T} .
$$

(4) Global objective optimization

Cloud data center virtual machine network flow optimization is mainly to solve the following problems. The first is to ensure that the traffic bandwidth of different communication levels can be maximized. The second is to maximize the utilization of link resources. Ensure that the use of the entire storage network reasonably, without local link traffic caused by excessive congestion or no-load. Let $\left(M_{i j}\right)_{T \times T}$ be a short form as $M$.

$$
\begin{gathered}
M a x M=a M_{1}+b M_{2}+c M_{3} . \\
\operatorname{MaxHG}(g)=-\sum_{k=1}^{W} G_{k} \ln G_{k} . \\
\left\{\begin{array}{l}
\sum_{m=1}^{3} M_{m, i j}=B_{i j}\left(M_{m, i j} \geq 0, B_{i j} \geq 0\right) \\
W_{i j} \geq \sum L_{i j}\left(L_{i j} \geq 0, W_{i j} \geq 0\right) \\
G_{k}=\frac{W}{G} \leq 1 \\
\operatorname{Max} F(g)=\alpha M+\beta H G(g) .
\end{array}\right.
\end{gathered}
$$

The parameters $a, b, c$ in Eq. 4 are three kinds of communication level weights. Eq. 5 uses the Shannon entropy to measure the equilibrium degree of link traffic in the network. In the constraint conditions of Eq. 6, firstly, the sum of the data link flows with different communication levels is described and secondly, the maximum bandwidth of the physical link is greater than the sum of the traffic that is equal to the sum of the traffic through the link. Eq. 7 is a harmonic function which is defined by the linear weighting method, which is converted to a single objective problem.

\section{Composite Particle Swarm Optimization Algorithm for Network Traffic Scheduling}

Particle Swarm Optimization algorithm is a bionic algorithm proposed by Kennedy and Eberhart in 1995.Through the imitation of a co evolution between species within different populations, namely from the stochastic solution of the iterative search for the optimal solution. Since the algorithm has the advantages of easy implementation, high accuracy and fast convergence speed, it has been widely used. Although the PSO algorithm can be directly applied to solve the problem of network traffic scheduling, but in the case of 
large-scale deployment of multi-node, there is an amazing amount of randomly generated number data links because of plenty of nodes, resulting in large dimensional vector that the algorithm will easily fall into local optimal solution while obtaining the global optimal solution. So we design the composite particle swarm optimization algorithm for network traffic scheduling to solve this problem. Have the amount of directed connections between network nodes as a solution to the spatial dimensions, and each dimension, whose value is assigned to the connection request flow, corresponds to a directed link. The D-dimensional vector X each particle corresponds, represents a solution to the system in accessing a resource allocation. The fitness function is the Eq. 7. All the variables in the solution vector are integer variables. The updating formulas for velocity and displacement vectors are shown below:

$$
\begin{aligned}
& v_{k+1}=\omega v_{k}+c_{1}\left(\text { pbest }_{k}-x_{k}\right)+c_{2}\left(\text { gbest }_{k}-x_{k}\right) . \\
& x_{k+1}=x_{k}+v_{k+1} . \\
& \omega=0.5+\frac{\text { rand }}{2}, \text { rand } \in(0,1) .
\end{aligned}
$$

$v_{k}$ is the velocity vector of the particle; pbest $t_{k}$ is the best solution for the particle itself; gbest $_{k}$ is the optimal solution for the whole particle swarm optimization; $\omega$ is the inertia weight coefficient; $c_{1}$ and $c_{2}$ are called "cognitive acceleration factor" and "social acceleration factor", which are generally $2 ; x_{k}$ is the position of the particle; $v_{k+1}$ is the sum of the $v_{k} ;$ pbest $_{k}-x_{k}$ and gbest $_{k}-x_{k}$ vectors, and the velocity of each particle is required to be within $v_{\max }$.

\subsection{Composite Particle Swarm Optimization Algorithm}

In order to improve the performance of the data center network's ability to select the best communication path, preventing optimal path search trapped in local optimal solution, this paper proposed the composite particle swarm network traffic scheduling algorithm, which upgraded the single particle swarm algorithm. For the previous section, the structure of the network cloud data center, divided a branch into a particle swarm, so the entire network is divided into multiple particles cluster solution space. The partition is shown in Figure 2:

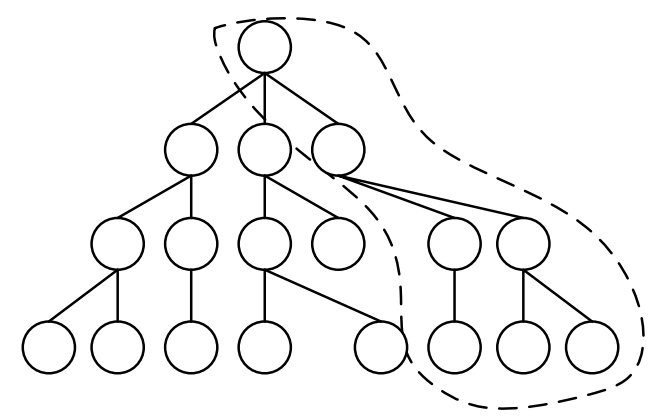

Figure 2. Particle Swarm Solution Space Division 


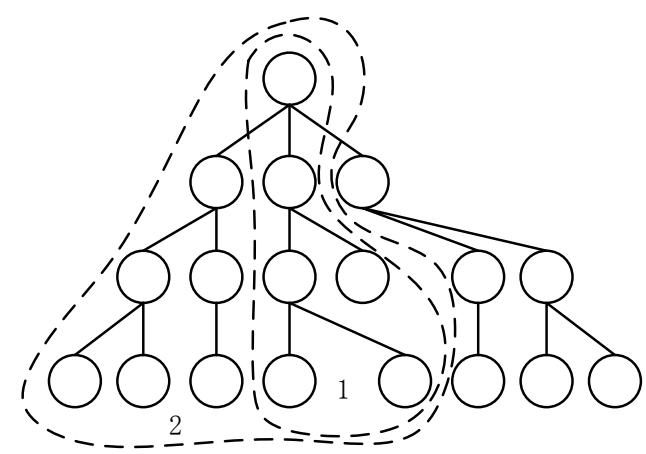

\section{Figure 3. Particle Swarm Solution Space Divided in Different Ways}

This way of division makes the original large solution space into some equivalence subspaces, and particles swarm optimization algorithm in each equivalence subspaces of exhaustive searches to find out each subspace of the optimal solution. Finally, the particle swarm exchange information with each other, so as to determine the global optimal solution, namely the network link optimal path.

The overall storage network has different ways to divide solution space, which can be divided as Figure2, and also can be divided as Figure3.The size of each solution space can be divided according to the different sizes of the network topology.

Composite particle swarm optimization algorithm proposed by this paper, is to establish multi particle swarm. It usually set 2 layers or 3 layers and the solution space is different for each layer. The first layer is divided into uniform, so that the algorithm can get the optimal solution quickly. The second and third layers are taken randomly search method and will obtain an optimal solution, compared with the first layer of the optimal solution. If it is better than the first layer of the optimal solution, then use the second or third layer of the optimal solution for network traffic distribution. The main process is shown in Figure 4.

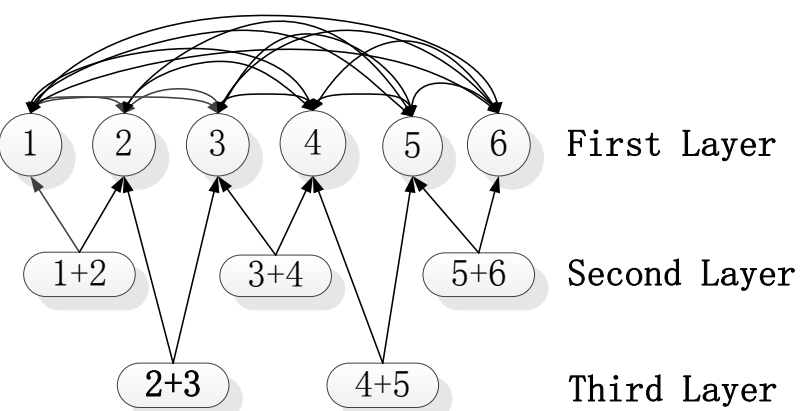

Figure 4. Multilayer Particle Swarm Optimization Algorithm

Assuming that the cloud data center network space traffic vector has $X$ contains a $N$ subspace, and each subspace connection dimension is $X_{i}$, which can be derived $X=\left[X_{1}, X_{2}, \cdots, X_{i}, \cdots, X_{N}\right]$. In the composite particle swarm optimization algorithm, if using the method shows in Figure 4, the first layer need construct $N$ particle swarm. The second and third floors respectively establish $N / 2$ particle swarm, so the algorithm needs to construct $2 N$ particle swarm. $2 N$ particles flow vector in the swarm take random value, the first layer has $X_{L 1}=\left[X_{1 \text { rand }}, X_{2 \text { rand }}, \cdots, X_{\text {irand }}, \cdots\right]$, the second layer has $X_{L 2}=\left[X_{1+2 \text { rand }}, X_{3+4 \text { rand }}, \cdots, X_{i+(i+1) \text { rand }}, \cdots\right]$, and the third layer 
has $X_{L 3}=\left[X_{2+3 \text { rand }}, X_{4+5 \text { rand }}, \cdots, X_{(i-1)+\text { irand }}, \cdots\right]$. After the first iteration, each layer will produce an optimal solution, then the first layer will exchange information with the second and third layer. If $X_{1+2 b e s t}$ is better than $X_{1 b e s t+2 b e s t}$, then the optimal solution will change and the best flow passage for particle swarm 1 and particle swarm 2 is $X_{1+2 b e s t}$, and so on. In practical application, the algorithm not only can obtain the global optimal path of the network, but also can obtain the optimal path between each partition. After the completion of the iteration, the system will have iterative detection threshold whether it is less than the limit, or attain the maximum number of iterations, so as to determine the continued iteration or retain the best quality, until the next issue network scheduling request to calculate.

\subsection{Network Traffic Scheduling Algorithm}

In this paper, a network traffic scheduling algorithm based on composite particle swarm optimization algorithm (MMPSOSA) is designed. The basic process is as follows:

a) To determine the network topology of cloud data center, including the basic situation of the network link. Determine the solution space vector according to the network topology, and then according to the topology of the network, divide the whole network into $\mathrm{N}$ sub network space, so as to construct three layers' particle swarm.

b) To confirm the size of particle swarm and fixed parameters, including the inertia weight $\omega$, cognitive acceleration factor $c_{1}$, social acceleration factor $c_{2}$, particle velocity threshold $\mathrm{v}$, maximum iterative $\mathrm{n}$ and iteration termination threshold.

c) Iterative search for each layer of particle swarm optimization to obtain the optimal value.

d) Compared with the optimal value of the first level using the optimal value of the second level and the third level, if the iterative results are better, exchange the optimal value.

e) After comparing the optimal value of each layer, the optimal flow solution vector is the optimal value under the current host node, and the data link is selected according to the optimal solution.

f) After the iteration, if it reaches a maximum number of iterations according to the judgment condition, terminate the iteration. At this time, the solution is the optimal solution. If the number of iterations is less than the threshold, continue to (c), until obtain the optimal value.

\section{Simulation and Evaluation}

Simulation experiment uses the CloudSim simulation platform.

During the process of this experiment, we regard the network bandwidth as virtual machine resources. So we convert the problem of network traffic scheduling into the problem of VM resources scheduling via MMPSOSA. Based on overloading the class Cloudlet, the core pseudocode are as follows:

public void Sim(Int num_user,Int VMCount,Int Bl)

\{

// Initialize

Calendar calendar=Calendar.getInstance();

boolean trace_flag=false;

CloudSim

CloudSim.init(num_user, calendar, trace_flag); //Initialization of packet

// Create data centers and agents

DataCenter datacenter=createDatacenter("Datacenter_0"); 


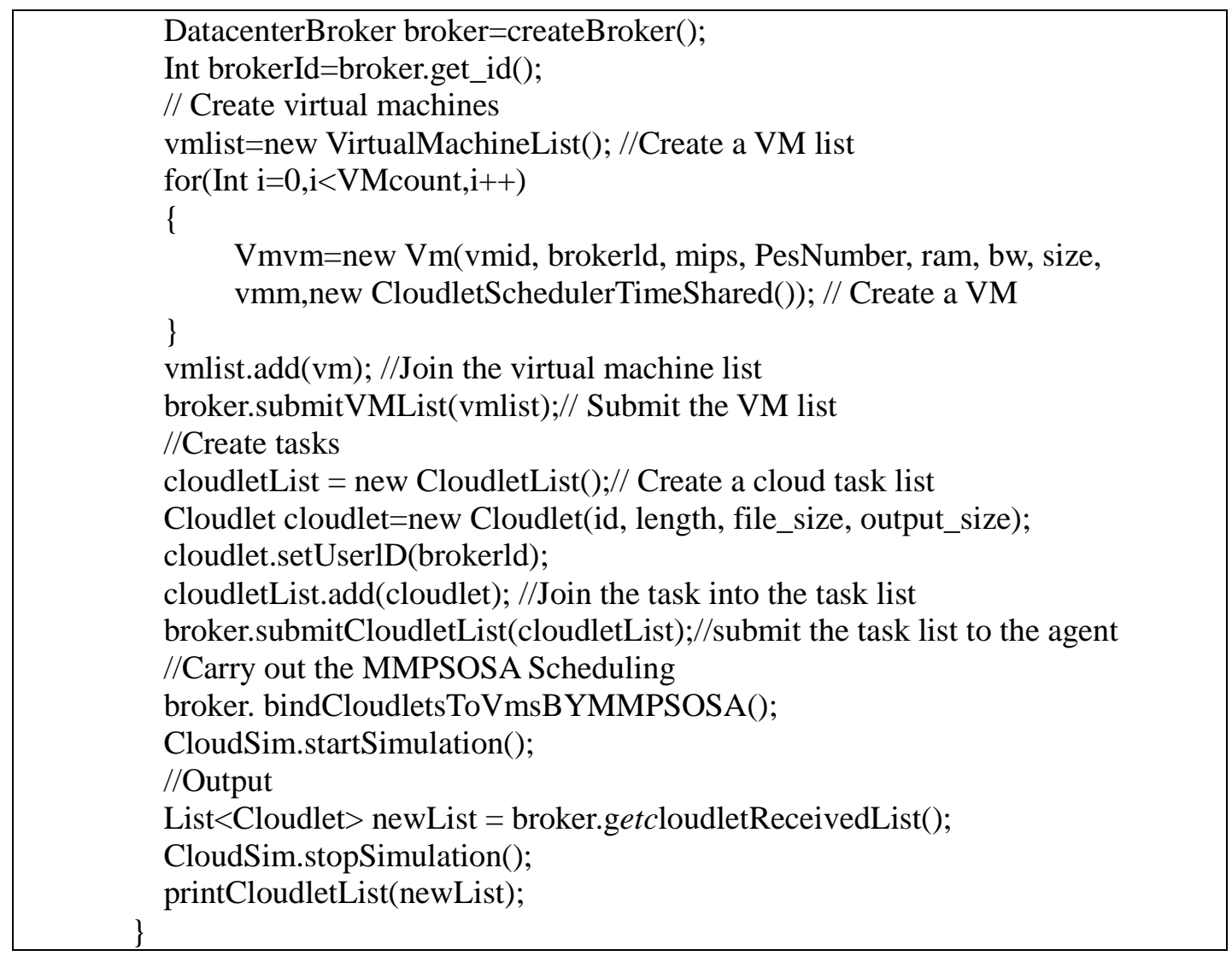

The simulation experiment chooses different sizes of tree network, including medium scale networks (64 nodes), large scale networks (256 nodes). The network link bandwidth is $1000 \mathrm{M} / \mathrm{S}$. In the experiment, the network traffic between each virtual host node is randomly generated. The maximum value is no more than $10 \mathrm{MB} / \mathrm{s}$ and the ratio of data flow to different QoS communication level is $S_{1}: S_{2}: S_{3}=1: 1: 1$. Experiment time is 180 seconds.

The experiment focuses on two aspects. The first one is the ability of the model in traffic scheduling, namely under two different kinds of network scales, the condition of the bandwidth and network link resource utilization of different levels using the algorithm of greedy bandwidth allocation, Genetic, ACO_and the algorithm in this paper. The second one is to investigate the convergence performance of the two layer multi particle swarm optimization algorithm with respect to the standard particle swarm optimization algorithm. The experimental resultsare is shown in Fig .5 and Fig .6 which compared with greedy network bandwidth scheduling algorithm, Genetic and ACO in terms of network scheduling. 


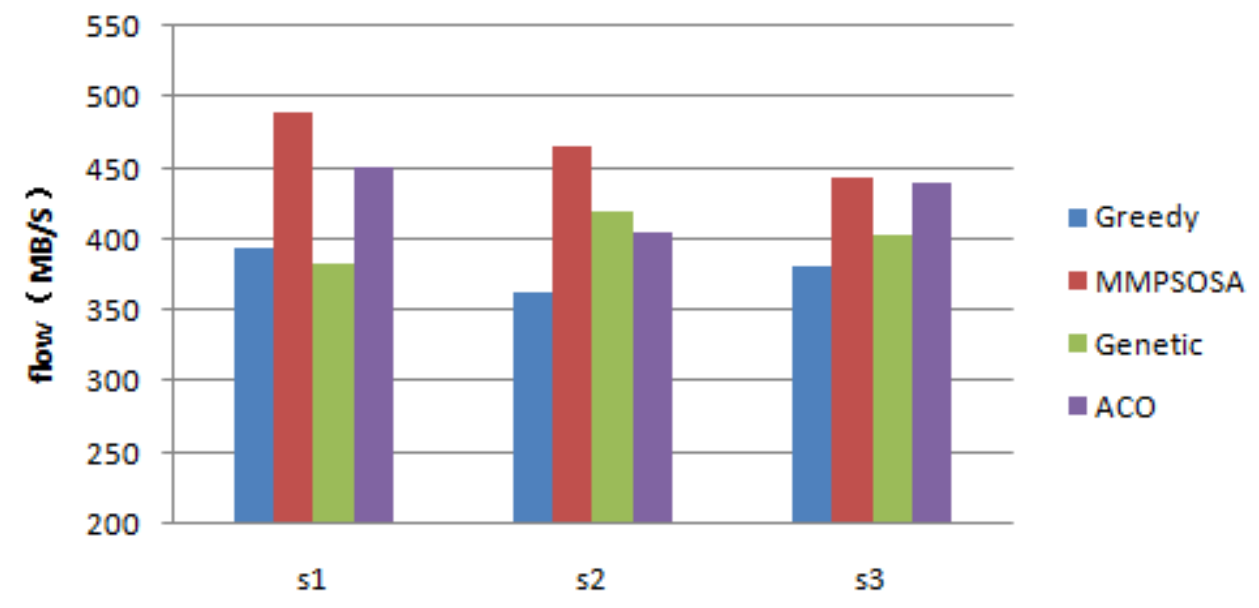

Figure 5. Different Communication QoS Level Traffic under 64 Nodes

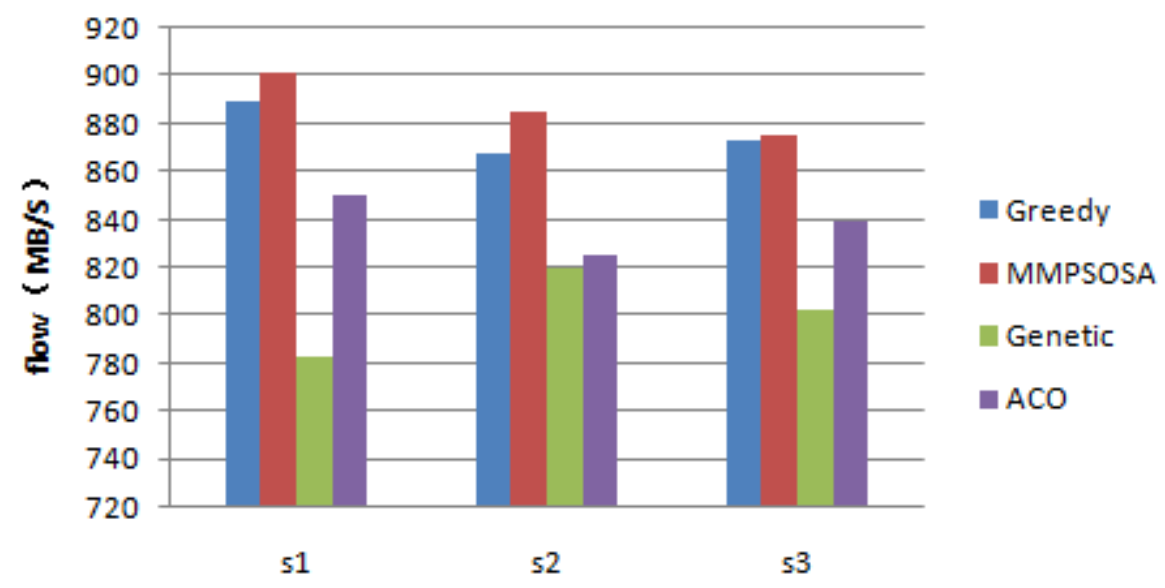

Figure 6. Different Communication QoS Level Traffic under 256 Nodes

The figures above show that composite particle swarm network traffic scheduling algorithm can be well controlled at all levels of QoS data traffic, which makes $S_{1}$ with higher level communicate firstly and reduce the traffic of $S_{2}$ and $S_{3}$ with lower level. In contrast, greedy network bandwidth scheduling algorithm, Genetic and ACO for the control of three kinds of QoS data traffic are much poor. The link utilization ratio of each network bandwidth scheduling algorithm under different virtual machine nodes is shown in Figure 7. The results of iterative performance are shown in Figure 8 which compared with the traditional PSO algorithm in terms of the number of iterations. 


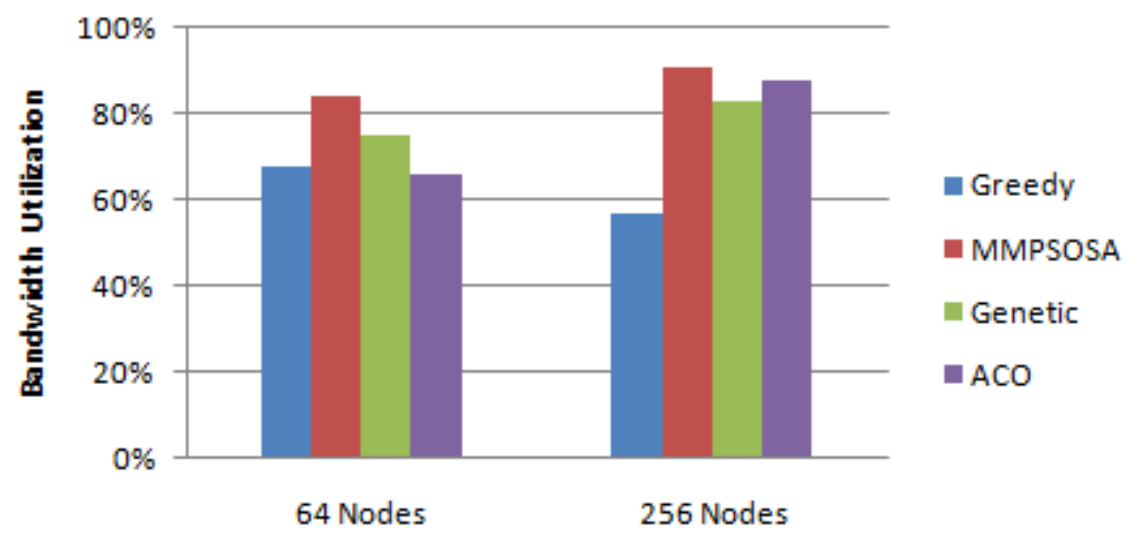

Figure 7. Link Utilization under 64 Nodes and 256 Nodes

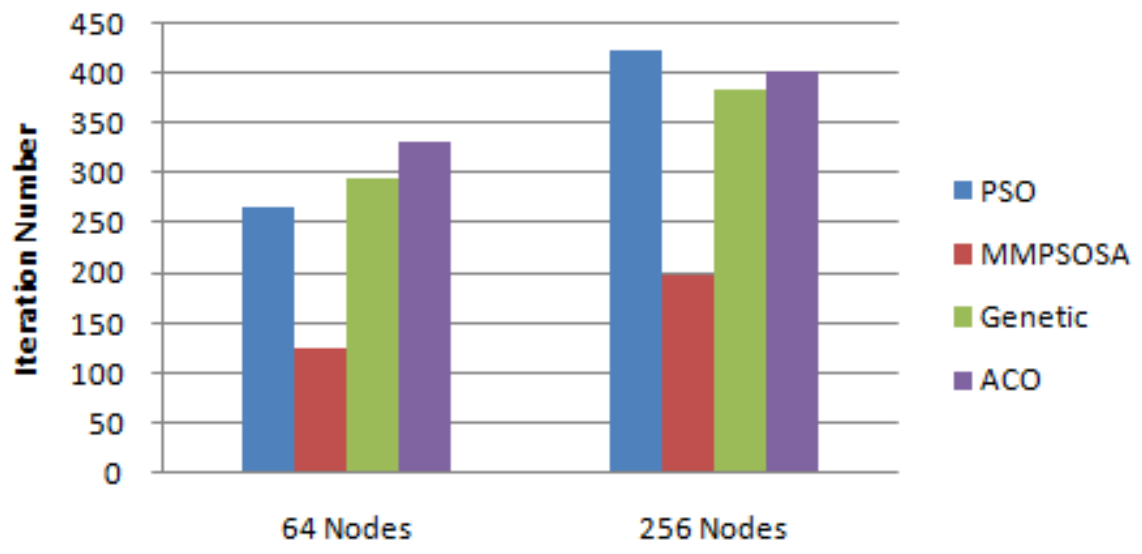

Figure 8. Algorithm Iterations at different Scale Network

In Figure 7, we can see that the composite particle swarm optimization algorithm for network traffic scheduling algorithm is higher than the greedy algorithm, Genetic and ACO in the network link usage. Especially in the case of virtual hosts doubled the number of nodes, the composite particle swarm network traffic scheduling algorithms on link utilization is increased, which is shown that this algorithm can be competent in dealing with large scale virtual hosts.

In Figure 8, it can be seen that if we use the traditional PSO algorithm, when the network scale expends and the number of links increase, the number of iterations will have exponential increase in size; and for the algorithm MMPSOSA, though the number of iterations will also increase, the basic maintain linear growth. The experiment shows that the particle swarm optimization algorithm can effectively reduce the convergence to the optimal value of the particle swarm optimization by dividing the original solution space into a number of equivalent subspaces.

The experiments above show that this design for multi-QoS network traffic between virtual machines scheduling model and scheduling algorithm can fully utilize network bandwidth resources, improve system resource utilization and balance so as to protect the business flow of different levels.

\section{Conclusions}

Aiming at solving the problems of unbalanced cloud data center virtual host network link bandwidth utilization and multi QoS (Quality of Service) security issues in different services, in this paper, a multi QoS based network traffic scheduling model for virtual 
machines is proposed. The optimization goal of model is to guarantee the requirements with different levels of data synchronization performance, and to realize the maximized utilization of inter VM network link bandwidth resources. Combined with the characteristics of hierarchical network structure, we put forward a network traffic scheduling algorithm based on particle swarm composite. By dividing the original solution space into several equivalent subspaces, each particle swarm is independently searched in the subspace. Therefore, the algorithm has a faster convergence rate and avoids falling into the local optimal value problem easily caused by the traditional algorithm. The simulation results show that the model and algorithm can improve the network link bandwidth utilization and guarantee the synchronization requirements of high priority data. The algorithm can not only be applied to the cloud data center network traffic scheduling and data security which are based on virtualization, but also can be used to schedule the flow of traditional distributed multi data centers to improve the quality of data exchanging.

\section{Acknowledgements}

This work was supported in part by Jilin provincial development and Reform Commission China (2014Y113).

\section{References}

[1] P. Rygielski and S. Kounev, "Network Virtualization for QoS-Aware Resource Management in Cloud Data Centers: A Survey”, Praxisder Information sverarbeitung und Kommunikation, vol. 36, no. 1, (2013), pp. 55-64.

[2] R. Jain and S. Paul, "Network virtualization and software defined networking for cloud computing: a survey", Communications Magazine, IEEE, vol. 51, no. 11, (2013), pp. 24-31.

[3] Y. Zheng-Kun, W. Qing-Bo, T. Yu-Song, W. Chun-Guang and R. Ni, "The Study of Algorithms for Multi-VMs Bandwidth Guarantee in Data centers", 2013 3rd International Conference on Computer Science and Network Technology, (2013), pp. 767-771.

[4] H. Ballani, D. Gunawardena and T. Karagiannis, "Network Sharing in Multi-Tenant Data centers", MSR, Tech. Rep. MSR-TR-2012-39, (2012).

[5] Y. Yue-quan, C. Zhi-qiang, T. Min and Y. Jian-qiang, "Fairness and dynamic flow control in both unicast and multicast architecture networks", IEEE Transactions on Systems, Man, and CyberneticsPart C: Applications and Reviews, vol. 37, no. 2, (2007), pp. 206-212.

[6] C. Jeong-Woo and C. Song, "Utility max-min flow control using slope-restricted utility functions", IEEE Transactions on Communications, vol. 55, no. 5, (2007), pp. 963-972.

[7] M. S. Abdulla and S. Bhatnagar, "Network flow-control using asynchronous stochastic approximation", Proceedings ofthe 46th IEEE Conference on Decision and Control, CDC, New Orleans, USA, (2008), pp. 5857-5862.

[8] F. Paganini, "A global stability result in network flow control[J]. Systems and Control Letters, vol. 46, no. 3, (2002), pp. 165-172.

[9] J. Young-mi and G. Kesidis, "Charge sensitive and incentive compatible end-to-end window-based control for selfishusers", IEEE Journal on Selected Area in Communications, vol. 24, no.5, (2006), pp. 952-961

\section{Authors}

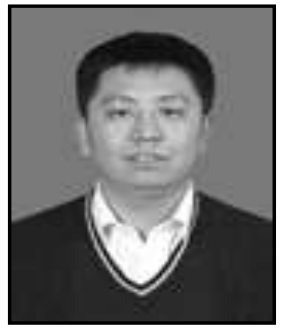

Huan Wang, He graduated from Changchun University of Science and Technology in 2009 and receive master degree from Changchun University of Science and Technology in 2012.In 2014, he was admitted to the school of computer, Changchun University of Science and Technology, studying for a doctorate. His research interests include network information security.

创新性说明 Innovative description: A multi QoS based network traffic scheduling model for virtual machines is proposed. In order to achieve the global optimal network traffic scheduling, the model uses Shannon information entropy to 
measure the network link traffic balance and uses the linear weighted sum method to transform the multi objective optimization into a single objective problem. At the same time, we put forward a network traffic scheduling algorithm based on particle swarm composite. By dividing the original solution space into several equivalent subspaces, each particle swarm is independently searched in the subspace. Therefore, the algorithm has a faster convergence rate and avoids falling into the local optimal value problem easily caused by the traditional algorithm. 
International Journal of Future Generation Communication and Networking Vol. 10, No. 1 (2017) 